\title{
VIVIR Y TRABAJAR EN FINCAS, FÁBRICAS Y \\ TALLERES. EXPERIENCIAS DE PADECIMIENTOS \\ Y CUIDADOS DE MUJERES MIGRANTES EN \\ MENDOZA Y BUENOS AIRES
}

\section{TO LIVE AND TO WORK IN FARMS, FACTORIES \\ AND WORKSHOPS. MIGRANT WOMEN'S \\ EXPERIENCES OF ILLNESS AND CARE IN \\ MENDOZA AND BUENOS AIRES.}

María Florencia LinardelLI ${ }^{*}$ - Alejandro GoldberG ${ }^{* \star}$

Resumen: En las últimas décadas se aprecia un aumento de investigaciones abocadas a la participación de las mujeres en los flujos migratorios. Incluso se afirma que asistimos a un proceso de "feminización de las migraciones" que implica el aumento de la cantidad de mujeres involucradas en los movimientos poblacionales de manera autónoma y la visibilización de su participación histórica. En ese contexto, ha cobrado relevancia dentro de las ciencias sociales la relación entre migraciones protagonizadas por mujeres y la salud/enfermedad/cuidado. El artículo propone un diálogo entre dos casos de estudio etnográficos situados en Argentina: 1) mujeres migrantes que trabajan en la agricultura en Mendoza; y 2) migrantes bolivianas insertas en talleres textiles de Buenos Aires. Mediante un enfoque comparativo exploramos las condiciones en

* Instituto de Ciencias Humanas, Sociales y Ambientales, Consejo Nacional de Investigaciones Científicas y Técnicas, CCT Mendoza.

** Instituto de Ciencias Antropológicas, Facultad de Filosofía y Letras, Universidad de Buenos Aires - Consejo Nacional de Investigaciones Científicas y Técnicas 
que acontecen sus trayectorias migratorias y los modos de vidal vivienda/trabajo en la sociedad a la que migraron, sus narrativas del padecimiento y las estrategias de cuidado que despliegan para recuperar la salud.

Palabras Clave: mujeres migrantes; salud/enfermedad/cuidado; trabajo agrícola; talleres textiles.

Abstract: In the last decades, there has been an increase in research on female participation in migratory flows. In fact, the research considers that the increase in the number of women involved in the population movements and the visibility of their historical participation configures a process of "feminization of migrations". In this context, the relation between female migrations and health/ disease/care also became relevant for the social sciences. This article proposes a dialogue between two ethnographic study cases located in Argentina: 1) immigrant women who work in agriculture in Mendoza; 2) Bolivian women immigrant workers inserted in textile workshops in Buenos Aires. Through a comparative approach, we explore the conditions in which their migratory trajectories and the life/housing/work modes take place in the society to which they migrated, their narratives of illness and the care strategies they deploy to recover their health.

Key words: migrant women; health/illness/care; agricultural work; textile workshops.

\section{INTRODUCCIÓN}

En las últimas décadas se aprecia un incremento en las investigaciones abocadas a la participación de mujeres en los flujos migratorios, vinculado con un proceso de "feminización de las migraciones", que implica no sólo el aumento de la cantidad de mujeres que forman parte de estos movimientos poblacionales de manera autónoma, sino también la visibilización de su protagonismo histórico (Magliano \& Domenech, 2009). En ese contexto, también cobró relevancia la relación entre procesos migratorios de mujeres y la salud, la enfermedad y los cuidados.

Partiendo del marco anterior, en este artículo proponemos poner en diálogo hallazgos de dos estudios de carácter etnográfico 
sobre migraciones y salud situados en contextos muy diferentes de Argentina: 1) mujeres migrantes que trabajan en fincas y fábricas del agro de la provincia de Mendoza; y 2) migrantes bolivianas que trabajan como costureras en talleres textiles de Buenos Aires. Desde un enfoque comparativo, analizamos los modos de vida/ vivienda/trabajo de estas mujeres en la sociedad a la que migraron y su relación con la salud/enfermedad/cuidado. Abordamos, entre otros, los siguientes interrogantes: ¿Cuáles son las condiciones en que viven y trabajan las migrantes que se insertan en la agricultura en Mendoza y en talleres textiles clandestinos de Buenos Aires? ¿De qué manera tales condiciones se relacionan con enfermedades y padecimientos? ¿Qué tipo de estrategias de cuidado despliegan para preservar/recuperar la salud — propia y de sus grupos domésticosen dichas circunstancias? Las respuestas que aproximamos se apoyan especialmente en las narrativas de las mujeres entrevistadas sobre sus experiencias cotidianas de padecimientos y cuidados.

Las preguntas formuladas resultan relevantes en un país como Argentina en el cual las mujeres migrantes originarias de países limítrofes y de Perú, generalmente, tienen como opción laboral empleos precarios y mal remunerados (Magliano, 2009). Esto se hace evidente en los dos casos que abordamos en este trabajo: tanto en el agro, como en los talleres textiles, las migrantes ocupan las escalas salariales más bajas, en los trabajos con mayor inestabilidad, y en condiciones de informalidad. Además, frecuentemente, talleres textiles y fincas constituyen el recinto de trabajo y de vivienda simultáneamente. Junto con ello, las extensas jornadas laborales y la doble jornada de trabajo (productivo y reproductivo) incrementan los riesgos de accidentes y los padecimientos de distinto tipo. Cabe destacar que en ambos casos las trabajadoras también enfrentan un limitado acceso a los servicios sanitarios debido a su condición de migrantes. En conjunto, sus derechos se encuentran con frecuencia vulnerados, a pesar de estar garantizados por leyes vigentes en el país ${ }^{1}$.

Para abordar el tema propuesto organizamos el artículo en tres secciones. En la primera, presentamos la perspectiva teórica utilizada. En la segunda, desarrollamos la metodología de ambos

1 Nos referimos a la Ley Nacional de Migraciones ( $\left.\mathrm{N}^{\circ} 25.871\right)$; a la Ley de Protección Integral para prevenir, sancionar y erradicar la violencia contra las mujeres ( $\left.\mathrm{N}^{\circ} 26.485\right)$; al Régimen de Trabajo Agrario (Ley $\mathrm{N}^{\circ} 26.727$ ); finalmente, a la Ley Nacional contra la Trata de Personas $\left(\mathrm{N}^{\circ} 26.842\right)$. 
estudios. En la tercera, exponemos los resultados. Analizamos las particularidades de los modos de vida/vivienda/trabajo en talleres textiles, fincas y fábricas, y abordamos las narrativas de padecimiento y cuidado relatadas por las migrantes. En las conclusiones señalamos aportes y limitaciones del enfoque propuesto y perspectivas futuras de la temática de investigación.

\section{PERSPECTIVA TEÓRICA}

En este trabajo recurrimos a herramientas conceptuales provenientes de dos campos: la antropología de la salud y los estudios de género y feministas. Para la antropología de la salud, los procesos vinculados con la enfermedad, los padecimientos y las estrategias de atención no se limitan a un fenómeno individual y biológico. Por el contrario, la perspectiva antropológica incorpora interrogantes sobre la subjetividad y la cotidianidad, preguntándose por las especificidades que cobran las experiencias cotidianas de las/los sujetos y el impacto de las mismas en los cuerpos y la salud de las personas, indagando asimismo en las formas de sufrimiento social y padecimientos que son consecuencia de esa cotidianidad (Grimberg, 2009; Goldberg, 2010).

Las emociones modelan la percepción del cuerpo, de la enfermedad y del dolor, y cumplen un rol central en las vivencias y proyecciones simbólicas realizadas por las/los sujetos (Scheper-Hughes y Lock, 1987), por cuanto constituyeron un elemento central desde el cual se analizaron las narrativas de padecimiento de las migrantes. En tal sentido, muchas de las situaciones por las que atraviesan estas mujeres - como las violencias de distinto tipo que sufren: estructural, física, simbólica y sexual- pueden impactar emocionalmente de forma negativa en su salud, debilitando el sistema inmunitario y aumentando así los riesgos de contraer padecimientos y enfermedades diversas. Así, las experiencias de violencia y las desigualdades constituyen aspectos que se corporifican en las y los sujetos (Csordas, 1994).

Algunas categorías conceptuales feministas posibilitaron comprender las relaciones entre migraciones, trabajo y salud como procesos modelados por las desigualdades sexo-genéricas. Para la teoría feminista la diferenciación sexual no es un dato biológico o dado, sino una de las formas más extendidas de desigualdad social, arraigada en la interacción personal entre los sexos y en instituciones sociales básicas: la familia, estructuras económicas y políticas, la 
organización de la producción y de la reproducción (Benería, 1981). Además, la subordinación de las mujeres adopta formas diversas al vincularse con otros sistemas de dominación social, como la clase y la racialización (Davis, 2005 [1981]; Crenshaw, 2012).

Otro aporte teórico feminista es considerar como trabajo las actividades desarrolladas -mayoritariamente por mujeres- en los grupos domésticos para asegurar la reproducción biológica, social y cultural de las personas (Maure, Linardelli \& Anzorena, 2016). Los hogares no se sustentan sólo por el ingreso percibido en los salarios, sino que éste se combina con trabajo reproductivo para producir bienes y servicios necesarios para la subsistencia. El trabajo no se reduce, entonces, a la producción remunerada de mercancías, sino que incluye la producción realizada en los hogares, como un aporte económico decisivo de las mujeres a la reproducción social (Carrasco, 2011; Federici, 2013). Este tipo de labores también son conceptualizadas como trabajo de cuidados, destacando que tienen características propias no comparables con el trabajo mercantil, asociadas a los aspectos subjetivos/emocionales que comportan (Rodríguez Enríquez, 2012; Pérez Orozco, 2014; Pessolano, 2016).

Por último, los estudios feministas visualizan al género como un determinante en el proceso de salud/enfermedad/cuidado, que opera en forma conjunta con otros factores, como son los de clase, etnia y edad (Esteban, 2010). Distintas investigaciones muestran la manera en que las desigualdades sexo-genéricas existentes en materia de educación, ingresos y empleo producen una "morbilidad genérica diferencial" y limitan la capacidad de niñas y mujeres para proteger su propia salud (Burín, 1990; Krieger, 2003; Velasco, et al., 2006; Valls-Llobet, 2009; Tajer, et al., 2013). Asimismo, la doble jornada implica pesadas cargas de trabajo y esfuerzos excesivos que deterioran progresivamente la salud de las mujeres y condicionan las posibilidades de autocuidado (Brito, et al., 2012).

\section{NOTAS METODOLÓGICAS}

\subsection{Contexto de las investigaciones}

Los datos del trabajo de campo que aquí presentamos provienen de investigaciones desplegadas en contextos diferentes, pero con perspectivas próximas, lo que facilita el abordaje comparativo propuesto. 
Por un lado, la investigación realizada en Mendoza, se inscribe en el marco de una tesis doctoral en curso (iniciada en 2014) sobre experiencias de salud, enfermedad y cuidados de mujeres migrantes que trabajan en la agricultura, financiada por una beca de CONICET. Cabe destacar que en esta provincia la presencia creciente de migrantes originarios/as de Bolivia y del norte argentino se tornó típica en distintas etapas de la producción de vid, hortalizas y frutas desde mediados del siglo XX (Benencia, 2009). En las últimas décadas, se crearon nichos específicos de empleo para las mujeres en la agroindustria abocada a productos de exportación (Mingo, 2015). Así, la feminización del trabajo asalariado agrícola y de las migraciones tomaron cuerpo en Mendoza, expresada en el incremento sostenido de la participación de mujeres bolivianas en los flujos migratorios y en la mayor presencia de mujeres en tareas agrícolas (Mingo, 2015; Moreno \& Martínez Espínola, 2017). El material empírico que exponemos fue recogido entre 2014 y 2017 en la localidad agrícola de Ugarteche, situada en el oasis norte de la provincia de Mendoza, a 40 kilómetros de la ciudad capital. Esta localidad constituye un enclave de migración limítrofe e interna y mantiene una fuerte tradición agrícola en torno a los cultivos de vid, frutales y hortalizas (Moreno \& Torres, 2013).

Por otro lado, las investigaciones etnográficas llevadas a cabo en talleres textiles en Buenos Aires, se desarrollaron desde 2008 en adelante, en el marco de distintos proyectos financiados por organismos de ciencia y tecnología, así como por ministerios públicos nacionales de la República Argentina. Considerando que se trata en la mayoría de los casos de unidades productivo-habitaciones ilegales, ingresar a un taller textil clandestino constituye una barrera infranqueable para cualquier persona que no tenga un vínculo familiar o laboral con el dueño del mismo. En tal sentido, la información primaria de este estudio proviene del trabajo de campo efectuado en otros dos ámbitos de indagación: 1) la Cooperativa textil 20 de diciembre - La Alameda, localizada en la esquina de Lacarra y Directorio (barrio de Floresta-CABA), integrada en buena medida por ex trabajadoras bolivianas de talleres textiles clandestinos; y 2) mujeres migrantes bolivianas con tuberculosis (TB), internadas en el pabellón Koch del Hospital Muñiz de la CABA, cuyo último trabajo, previo a la internación, fue en uno de estos talleres. 


\subsection{Estrategias de recopilación, análisis y validación}

El abordaje metodológico de ambas investigaciones se enmarcó en el enfoque etnográfico, habiéndose priorizado, para cada una, estrategias cualitativas específicas. En el caso de las trabajadoras agrícolas migrantes, las técnicas de recolección de la información utilizadas fueron relatos de vida, técnicas observacionales con diferentes grados de participación y entrevistas a informantes institucionales. Los relatos de vida fueron recuperados mediante sucesivas entrevistas en profundidad a seis trabajadoras, seleccionadas mediante muestreo intencionado. Se realizaron observaciones en diferentes espacios comunitarios en los que participan trabajadoras de la localidad de Ugarteche: en los encuentros semanales de un grupo de tejido integrado por mujeres migrantes; en la feria de Ugarteche, espacio que constituye un lugar de trabajo habitual de algunas entrevistadas; y en instancias colectivas, como reuniones de líderes migrantes, espacios educativos y actividades recreativas de la comunidad.

En el caso de las migrantes que residieron y trabajaron en talleres textiles, se combinó el trabajo de campo (observación participante, entrevistas en profundidad semi-estructuradas, grupos de discusión y análisis de narrativas), con el trabajo con fuentes secundarias (recopilación y análisis bibliográfico, estadístico, normativo, etc.). Las unidades de análisis fueron mujeres bolivianas adultas, que trabajaran y/o vivieran (o hubieran trabajado/vivido) en talleres textiles clandestinos de Buenos Aires. Tanto las observaciones de campo como las entrevistas y los grupos de discusión realizados, se efectuaron fundamentalmente en centros de salud y cooperativas textiles del suroeste de la ciudad, integradas éstas últimas, en buena medida, por mujeres migrantes bolivianas ex trabajadoras costureras en los talleres ${ }^{2}$.

Por la diversidad de los datos de campo, el análisis se sustentó en la combinación de dos estrategias: análisis temático — sugerido para las investigaciones con relatos de vida- (Mallimaci \& Giménez Béliveau, 2006) y connecting strategies que sitúan los relatos en una estructura contextual para que tomen un sentido más amplio (Maxwell \& Miller, 2008). Las estrategias de validación utilizadas en ambos casos fueron la triangulación de métodos, que permite contrastar la información

2 Todas las actividades descriptas contaron con el consentimiento de las participantes. Además, los proyectos de investigación fueron evaluados por comisiones de CONICET, lo que supone la revisión de aspectos éticos. 
obtenida a través de una técnica con otras técnicas, y la triangulación de espacios y tiempos, que consiste en aplicar las técnicas de recogida de información en diferentes momentos y lugares, para evaluar la consistencia de los resultados (Álvarez, 2011).

\subsection{Mujeres participantes en el estudio}

En el caso de las mujeres migrantes de la localidad agrícola de Ugarteche, la selección de informantes siguió el criterio de maximizar las diferencias, buscando entrevistadas que expongan un rango amplio de experiencias individuales. Se entrevistó a mujeres de distintas edades —entre los 29 y los 57 años-, trabajadoras activas y una de ellas retirada, migrantes de mediana y de larga permanencia. Una de la entrevistadas es originaria del norte argentino (provincia de Salta) y las restantes provienen de Bolivia, de los departamentos de Cochabamba, Potosí y Chuquisaca. Un rasgo común de las mujeres es su origen campesino-indígena.

Las mujeres migrantes entrevistadas en Buenos Aires fueron adultas, de los 18 a los 35 años, solteras o en pareja, con o sin hijos/ as. En su mayoría, eran oriundas de zonas rurales: en primer lugar, del Departamento de La Paz y alrededores, seguidas por aquellas provenientes de Cochabamba y Santa Cruz de la Sierra, y en menor medida de Oruro. Antes de migrar, buena parte de ellas trabajaba en tareas agrícolas, mientras otras lo hacían en el comercio informal y en la limpieza, no contando con experiencia en el rubro de costura hasta su llegada a Buenos Aires (exceptuando algunas mujeres provenientes de La Paz con antecedentes en la actividad). El nivel de educación formal alcanzado con mayor frecuencia entre las entrevistadas fue la enseñanza media y, en menor medida, había casos de analfabetismo o que alcanzaron el nivel básico de educación.

\section{RESULTADOS}

\subsection{Contextos de vulnerabilidad social en destino: desigualdades de clase y sexo-genéricas y modos de vida/ trabajo/vivienda precarios}

Según datos de la Organización Internacional para las Migraciones (2012), de las/los 700.000 sudamericanos/as que emigraron de sus 
países entre 2002 y 2012, 500.000 llegaron a la Argentina, encabezados por paraguayos/as y bolivianos/as. Por su parte, la Dirección General de Migraciones de la República Argentina calculó que entre 2004 y 2012 había aumentado notablemente la cantidad de ciudadanos/as bolivianos/as que solicitaron la residencia en el país: de los 10.496 permisos de residencia otorgados en 2004 se pasó en 2006 a 86.509. Es importante remarcar que las estadísticas oficiales no contemplan a las personas en situación de irregularidad migratoria, con lo cual el volumen de esta población, como mínimo, tendería a duplicarse en términos absolutos. Los datos disponibles exhiben que los/las ciudadanos/as bolivianos/as, ocupan el segundo lugar — detrás de los/las paraguayos/as - en el ranking de inmigrantes residentes en Argentina (INDEC, 2010).

El crecimiento de estos flujos migratorios intrarregionales coincide con profundas transformaciones en los modos de explotación del trabajo realizado por migrantes en el capitalismo global, que es posible observar tanto en países centrales como en los del sur del mundo. En cuanto al trabajo agrícola en América Latina, los procesos de reconversión productiva de las últimas décadas del siglo $\mathrm{XX}$, jugaron un papel destacado en las transformaciones migratorias. La expulsión de medianos y pequeños productores, la difusión creciente del trabajo asalariado y la precarización del empleo rural se acompañaron de continuas migraciones campo-ciudad o a través de las fronteras (Teubal, 2001). En este contexto, comenzó a registrarse el aumento de emigración rural femenina y del trabajo asalariado de mujeres en el agro (Arizpe \& Aranda, 1981; Teubal, 2001). La reconversión productiva de la agricultura y su inserción en el mercado internacional se sostuvo en una flexibilidad 'salvaje' de la mano de obra, que mantiene a grandes masas de trabajadores/as —especialmente mujeres, migrantes y jóvenes- en trabajos temporales, con largos períodos de desempleo y formas de trabajo sumamente precarias (Lara Flores, 1995). Estos procesos han tenido implicancias específicas para las mujeres migrantes trabajadoras de la agricultura, quienes padecen la condición de precariedad laboral del sector, a la vez que experimentan desigualdades sexo-genéricas que las afectan distintivamente: falta de cobertura en eventos reproductivos; violencia en el trabajo; asignación a los puestos peor remunerados y la invisibilización de sus calificaciones laborales para ciertas tareas por considerarlas habilidades femeninas "naturales" (Aparicio, 2012; Valdés Subercaseaux, 2015; Mingo, 2015). 
En Argentina, y específicamente en Buenos Aires, los talleres clandestinos de producción textil ligados al tráfico de personas, trata y reducción a la servidumbre, surgen y se dinamizan a partir de mediados de la década de 1990 (Goldberg, 2010; 2014 ; ; 2014; Benencia, 2009a; Lieutier, 2010; Colectivo Situaciones-Colectivo Simbiosis Cultural, 2011; Salgado, 2015; Miranda, 2017). En ese momento, debido a la aplicación del plan de convertibilidad y la consecuente sobrevaluación del peso, las industrias del sector de la indumentaria comenzaron a cerrar sus líneas productivas y a centrarse en la comercialización, actuando como importadores. Las principales empresas sustituyeron los eslabones nacionales por extranjeros, a partir de la subcontratación de terceros para las etapas de corte, bordado, costura, etc. y/o la importación de productos terminados. Esto agudizó la desarticulación del sector y permitió a las grandes marcas trasladar a los productores los bajos precios impuestos por la importación, centrando sus actividades en el diseño y la publicidad (Lieutier, 2010). Así, se combinaron dentro de la cadena de valor segmentos legales con otros clandestinossumergidos (Klein, 2001), como sucede con el caso de los talleres que se abordan en este trabajo. Al hablar de talleres clandestinos, entonces, nos referimos a aquellos que se encuentran en el extremo más alejado de la formalidad laboral y que, en buena medida, se nutren de mano de obra proveniente de la trata de personas con fines de explotación laboral (Salgado, 2015).

\subsubsection{El trabajo de mujeres migrantes en el agro mendocino}

Los relatos obtenidos en nuestro trabajo de campo nos permiten reconstruir algunos recorridos comunes en las experiencias laborales de las entrevistadas. Las primeras experiencias de trabajo en el contexto migratorio se vinculan con lo que se conoce como "trabajo golondrina”. Este momento constituye un punto crítico en cuanto a las condiciones de precariedad en el empleo. Se trata de actividades ligadas a una rápida movilidad territorial por diferentes provincias argentinas al ritmo de distintas producciones. Es típico de esta forma de empleo que las/los trabajadores/as residan en el lugar de la producción. Se trata de carpas o piezas que no cumplen con requisitos básicos de higiene ni de seguridad y que presentan características de hacinamiento y escasa protección de las inclemencias climáticas. Adicionalmente este período supuso para las mujeres la exposición a 
situaciones de violencia en el tránsito o en los lugares de alojamiento, debido a la falta de condiciones mínimas de seguridad personal:

[...] sabiamos vivir directamente en la chacra, donde está la zanahoria, allá en las orillas armaban las carpas [...] La comida la hacíamos ahí no más donde estábamos, en esa misma carpita donde dormíamos (Rosa, migrante salteña, 40 años).

Ahí vivíamos en una pieza chiquitita... todos dormíamos en el piso, tendíamos una colcha porque no teníamos colchones, nada... dormía en los pies de mi hermano, mi hermano dormía con su señora, con su hijito y yo dormía en sus pies... (Rita, migrante boliviana, 37 años).

Las entrevistadas relatan que, luego de períodos variables de tiempo en cada caso $^{3}$, lograron completar el ciclo de trabajo anual en la provincia lo que les permitió fijar su residencia en Mendoza. Dicho ciclo laboral se organiza por la conjugación de tres tipos de actividades: en fincas, en tareas de pleno campo; en fábricas de selección y empaque de fruta en fresco para la exportación y en el comercio en la feria local. Fincas y fábricas son identificadas por las trabajadoras como los espacios laborales más relevantes, tanto por el ingreso que les reportan, como por la extensión temporal de ese empleo en el calendario anual de trabajo. Se trata de espacios en los que se juegan condiciones de trabajo diferentes y que son significados de manera diversa por las trabajadoras. No obstante, presentan aspectos comunes, como la asignación de las mujeres migrantes a las tareas más precarias y la inestabilidad en el empleo.

Respecto del primer punto, tanto en fincas como en fábricas funciona eficazmente una división sexual y racial del trabajo que se hace visible en la asignación de las mujeres migrantes a los puestos más precarios y peor remunerados, y en la naturalización de sus calificaciones como trabajadoras, bajo el argumento de que constituyen habilidades femeninas naturales antes que productos de su cualificación (Lara Flores, 2003; Mingo, 2015). Así, las trabajadoras son reclutadas selectivamente para determinadas tareas según características que les atribuyen los empleadores: la supuesta mayor resistencia a trabajos de gran carga física que tendrían por ser bolivianas

3 Solo una de las entrevistadas no transitó por el empleo golondrina. Las demás mujeres entrevistadas permanecieron en este tipo de empleo entre cuatro y veinte años, realizando migraciones "pendulares" o “circulares” (Moreno, 2017). 
y la mayor responsabilidad y dedicación que tendrían como mujeres. Estas creencias, sostenidas en la culturización de relaciones de dominación históricas (Magliano, 2009) ocultan las pocas posibilidades que tienen las migrantes de acceder a empleos en mejores condiciones. La doble "valoración" dada por empleadores por ser mujeres y migrantes, no redunda en mejores condiciones de empleo, por el contrario, es usual en el agro mendocino que los puestos de trabajo exclusivamente masculinos correspondan a las tareas mejor pagadas y con posibilidades de acceso a un puesto permanente (Mingo, 2015).

En cuanto a la inestabilidad, aun cuando las trabajadoras laboran todos los años para el mismo empleador, se las contrata anualmente como trabajadoras temporarias, lo que redunda en menores derechos y protecciones sociales. Detrás del argumento de la gran variabilidad de la producción, cada temporada las mujeres desconocen si serán convocadas a trabajar y bajo qué condiciones, aspectos cuya determinación es atribuida por los empresarios al volumen de producción. Así, eventuales bajas en la productividad empresarial son pagadas por las mujeres con su desempleo.

Para finalizar, un elemento que resulta distintivo de las labores en fincas es la forma de pago. La remuneración "a destajo" redunda en jornadas extenuantes y cargas excesivas para lograr ingresos básicos (Lara Flores, 2003). Alcanzar un salario mínimo sólo se vuelve posible mediante una intensificación del trabajo, el empleo de la fuerza de trabajo de todo el grupo familiar o bien el aumento del tiempo de trabajo. Frecuentemente todas estas opciones en forma conjunta:

Nosotros hemos trabajado con mis hijos... llenábamos el tacho, lo echábamos al camión y te daban una ficha. Y yo veía que mis hijos ya tenían una bolsita así de fichas... dios mire... me faltaban manos para cosechar [...] yo llenaba los tachos y ellos venían y acarreaban [...] iban y corrían ¿usted sabe cómo corrían? Descansaban un ratito y le dábamos otra vuelta. No descansaban ni diez ni quince... yo seguía cosechando y llenando los tachos hasta que ellos descansen (Alba, migrante boliviana, 52 años).

\subsubsection{Los talleres textiles clandestinos}

A partir de los relatos de las protagonistas, fue posible dar cuenta del funcionamiento de las redes organizadas de tráfico ilegal de personas referidas con anterioridad, que abarcan desde el 
reclutamiento en origen por medio del engaño, pasando por la gestión del traslado, hasta la inserción en los talleres textiles clandestinos con fines de explotación de la mano de obra en condiciones análogas a la esclavitud (Goldberg, 2014a; 2014 ):

Le dijo a mi marido que ella nos podía conseguir trabajo, que se ganaba muy bien: 150 dólares (...) Ella nos dijo maravillas de Buenos Aires, del trabajo, como es el trato... y nos dijo que ella nos pagaría los pasajes (...) También nos dijo: como no tienen DNI van a hablar con unas personas que les van a hacer pasar... y pasamos con documentos "truchos": hay personas que se dedican a alquilar documentos, ellos te alquilan directamente. Ella ya sabía, ya conocía porque había hecho pasar a otras personas más, ellos están ahí en la frontera... No lleven nada de plata, dijo, que yo les voy a pagar todo (Entrevista a Ma, migrante boliviana, ex trabajadora en un taller textil clandestino de Buenos Aires, integrante de la Cooperativa textil 20 de diciembre-La Alameda).

Estos talleres constituyen una de las pocas opciones de empleo accesibles para las/os migrantes bolivianos/as, ya que garantizan trabajo y vivienda al mismo tiempo, sin necesidad de tener la documentación regularizada. Especialmente para las mujeres, quienes comenzaron a ser más demandadas debido a que, además de la costura, cumplen con tareas de limpieza y cocina que los empleadores —en su mayoría también bolivianos- les exigen.

[...] desde mi nacionalidad boliviana, el primer trabajo, el más accesible siempre es un taller clandestino. En el único lugar en el que no te piden documentos... No tenía otra oportunidad y tenía que mantener a mi familia así que decidi entrar a trabajar ahi porque era mi única opción... no tuve más alternativa (Entrevista a No., ex trabajadora en talleres textiles clandestinos).

La realidad referida se manifestaba cuando, durante la anterior gestión de gobierno nacional, se producían allanamientos judiciales en estos talleres ilegales, como consecuencia de las denuncias realizadas por La Alameda, la Defensoría del Pueblo de la CABA u otras instancias. Durante los procedimientos eran las/los costureros/ as quienes enfrentaban a la policía para evitar que confiscaran las máquinas y clausuraran el taller. Estas prácticas expresan que al interior del taller se perpetúan distintas relaciones de dominación, de violencia física y simbólica, de presiones y amenazas sutiles (y 
no tanto) hacia las/los trabajadores/as, quienes, ante la falta de conocimiento sobre las formas de trabajo en el país, y otro tanto por la falta de opciones y alternativas con las que cuentan para vivir, "aceptan" tales condiciones laborales de explotación y precariedad (Domínguez, 2010).

Sintéticamente, podemos decir que el modo de vida/trabajo/vivienda en estos talleres puede caracterizarse como precario, aunque algunas de las situaciones experimentadas por muchas de estas trabajadoras estarían más cercanas a una esclavitud servil: violencias de distinto tipo por parte del tallerista contra ellas y sus hijos/as; retención de los documentos; prohibición de salida autónoma del taller; alimentación deficitaria; entre 12 y 19 horas de trabajo; condiciones edilicias y ambientales de hacinamiento, escasa ventilación y mínima entrada de luz solar, tanto en el espacio donde están las máquinas como en el que duermen las trabajadoras y sus familias; instalaciones eléctricas defectuosas que representan un peligro latente de incendio, como sucedió en 2010 y 2015; falta de condiciones higiénicas y salubres en todo el recinto, especialmente en baños; constante aspiración del polvillo desprendido por la costura de la tela, incluso al dormir.

La extrema precariedad socio-laboral y habitacional descrita constituye, al mismo tiempo, un proceso estructural y una experiencia de vida en el marco de las trayectorias migratorias de estas mujeres, que provoca un proceso de desgaste permanente en su salud.

\subsection{Padecimientos, deterioro de la salud y estrategias de cuidado}

\subsubsection{Daños, padecimientos y estrategias de cuidado en contextos de trabajo agrícola}

Hasta aquí hemos visto que la creciente participación de las mujeres migrantes como asalariadas agrícolas no supuso necesariamente un cambio beneficioso en sus condiciones de vida, ya que - por ejemplo- el pago a destajo implica que obtener mejores ingresos sólo sea posible a costa del creciente desgaste físico. Abordaremos ahora la vinculación de estos procesos con las enfermedades, daños y padecimientos producidos por el trabajo, y señalaremos algunas estrategias de cuidado de la salud desplegadas por las trabajadoras. 
Respecto del primer punto, tal como muestran otras investigaciones (Camarena Ojinaga et al., 2013), las trabajadoras señalan como daños o enfermedades asociadas al trabajo en fincas y fábricas sintomatologías dermatológicas y respiratorias, debido al uso de agrotóxicos, pesticidas y desinfectantes. También manifiestan padecer dolores corporales diversos debido a posturas forzadas en las cintas de producción o por la intensa demanda física del trabajo. También describen la recurrencia de accidentes laborales y el impacto nocivo de temperaturas extremas. Una trabajadora relata:

Hay muchos accidentes, se desmayan, se resbalan, se rompen la cabeza, se golpean la columna y se desvian la columna... [se caen] por el durazno que hay en el piso, hay agua, lavandina, es muy resbaloso. Siempre tenemos que lavar con detergente, con ese... soda cáustica... con detergentes muy fuertes... te queman, se te pela toda la mano... por eso yo no quiero ir a la cinta [...] cuando entré a la cinta creo que se me hizo ese, de golpe apareció, pica, pica... es el ácido, un ácido fuerte que te dan para lavar... horrible te hace tu mano. La piel se hace bien finita, apenas que tocas ya se raja la mano [...] nos dicen que tenemos que usar guantes, pero los guantes no resisten, nos dan unos guantes descartables que no sirven, se rajan (Rita, migrante boliviana, 37 años).

La falta de horas de descanso es otro elemento referido por las trabajadoras como una de las condiciones que afectan su salud, aspecto se vincula estrechamente con la doble jornada. Cuando les pedimos a las trabajadoras que relaten un día habitual de trabajo manifiestan que, en general, su jornada inicia en la madrugada y se extiende hasta pasada la medianoche:

Yo dejaba a los niños míos que son cinco chicos y me iba a trabajar pasando el río [...] trabajaba ahí hasta las doce y venía ligero les daba de comer y les mandaba a la escuela. Terminaba de comer limpiaba mi casa y me iba corriendo a trabajar de vuelta [...] llegaba a las seis y ellos ya estaban acá. Cuando yo llegaba les hacía la comida en la tarde, en la noche hasta la una de la mañana yo lavaba la ropa. Me acostaba y me levantaba a las cinco de la mañana y cocinaba. Mi diario era cocinar todos los días para ellos para que vayan a la escuela y mi esposo vaya a trabajar y yo vaya a trabajar (Alba, migrante boliviana, 52 años).

Las trabajadoras identifican que la combinación de todas estas labores las lleva a dormir menor cantidad de horas de las que 
estiman como necesarias. Consecuentemente manifiestan síntomas de fatiga recurrente y dolores corporales.

Por otra parte, la estricta vigilancia y el riguroso control del proceso de trabajo a los que son sometidas en el espacio de las fábricas, es referido por las trabajadoras como un padecimiento asociado a su empleo. Esto conduce a que algunas de ellas valoren el trabajo en fincas como un espacio de mayor libertad, en el que habría un menor control patronal que en el rígido espacio fabril. Asimismo, la finca se presenta como un ámbito de socialización donde pueden intercambiar con otras mujeres.

[en la fábrica] Te salís más cansada que ir a trabajar en la viña... en la viña caminamos, desbrotamos, pero estamos al aire libre [...] es tranquilo en la viña, a mí me gusta trabajar en la viña, charlas, conversas, haces bromas, escuchas música, te reís, tranquilo en la viña. Pero acá pareces preso... no tenés que charlar, no tenes que jugar, no tenes con quien charlar porque yo estoy sola allá [...] te dicen que está filmando la cámara que enseguida le van a llamar al encargado, porque hay ahora todo cámara, para vigilar a la gente si está trabajando o no está trabajando... todo cámara. No puedes comer nada... prohibieron todo (Rita, migrante boliviana, 37 años).

La violencia en los espacios de trabajo, suele ser otro de los padecimientos destacados por las trabajadoras, causante de elevados niveles de angustia. Esta violencia se despliega en dos sentidos, por su condición de migrantes y personas racializadas y por su condición de mujeres en un espacio tradicionalmente masculino.

El hijo del patrón me llevaba a descargar la estufa. Cuando al bajarme me empezaba a manosear... y alguien le contó al patrón, yo tenía miedo de contarlo... por ahí me van a dejar sin trabajo decía yo... no hablaba, yo me callaba todo lo que me manoseaban, porque me daba miedo ir a trabajar con él, pero tenía que ir, porque yo necesitaba, sino ¿dónde iba a ir a trabajar? (Rita, migrante boliviana, 37 años).

Las enfermedades y padecimientos detallados, son abordados por las trabajadoras mediante diversas estrategias. La consulta a efectores de salud constituye una alternativa limitada por las condiciones de trabajo. La falta de registración les impide el goce de licencias por enfermedad o por cuidado de familiar enfermo, al tiempo que el pago por jornal implica que un día de reposo o 
dedicado a la asistencia de otro/a es un día sin remuneración; ello conduce a que las mujeres evalúen la conveniencia de la pérdida de este ingreso frente a una situación de enfermedad. La distancia entre los lugares de trabajo, los hogares y las instituciones sanitarias es aducida como una dificultad adicional.

No te entienden en la fábrica, por ahí decís ¿me pueden cambiar porque tengo turno mañana, tengo que llevar...? "No es mi problema, no sé qué harás, vos tenes que cuidar tu trabajo no yo, yo llamo a reemplazo y listo (Rita, migrante boliviana, 37 años).

Los límites, y en ocasiones imposibilidades, para acceder a servicios de atención médica tornan relevantes las estrategias de cuidado desarrolladas por las trabajadoras para afrontar situaciones de enfermedad. Estas prácticas, entendidas por la antropología en términos de autoatención, refieren a aquellas actividades cotidianas que se desarrollan en los grupos domésticos sin intervención de curadores profesionales (Menéndez, 2005).

Plantea Sonia (migrante boliviana, 35 años) respecto de las dificultades para acceder a turnos pediátricos: "yo sé cómo puedo curarle [a su hijo], puedo comprar medicamentos, mi hermana me puede ayudar porque es enfermera". Alba, por su parte, refiere que evita acudir a hospitales o centros de salud, ya que le provocan temor y las asocia con el encierro antes que con el cuidado de la salud:

[...] no me gusta ir a la sala, no me gusta ir a ningún lado. O sea, no estoy enferma ¿Para qué voy a ir? [...] lo que pasa es que es muy difícil que los bolivianos estemos enfermos, porque comemos cosas muy sanas y no nos enfermamos... como trigo y esas cosas que producíamos en Bolivia, también papa ¿vio? Hasta ahora yo hago esas comidas típicas bolivianas. Yo mucho argentino no sé. Yo me cocino típico boliviano [...] mi nieta come todo lo que hago y hasta el día de hoy jamás cayó en el hospital, ella no se enferma. [...] me da miedo el hospital, me da mucho miedo, porque ya he pasado por muchas cosas, con mi mami y con mis hijos, entonces cuando voy al hospital... haga de cuenta que estoy entrando a una cárcel, que no voy a salir más.

Rosa, en cambio, manifiesta que sí realiza consultas médicas, pero que debe organizarlas de acuerdo a los períodos de trabajo en la finca: En la temporada del desbrote, de la viña que hago, casi que no la ocupo la obra social. Porque no voy, no tengo tiempo. Claro, si 
llego de trabajar a la noche. $Y$ directamente no voy [...] O puede ser que alguna urgencia, pero casi no voy. Solamente yo hago los estudios cuando termino la fábrica, ahí hago mis estudios.

María (migrante boliviana, 57 años) afirma "yo misma me trato de sanar". Ella ejerce la medicina campesina desde su infancia, cuando aprendió de sus padres y sus abuelos distintas prácticas de curación. Sin embargo, también asiste al centro de salud para realizarse controles médicos y, si es necesario, sugiere a quienes le consultan que acudan al hospital. María atiende a las personas de su comunidad frente al susto, el estómago volcado, torceduras o quebraduras.

Todo eso, yo hago con mi mente que me viene, la idea nos lleva. A mí me da lástima el dolor que tiene, son dolorosas las lastimaduras. Y por eso, pongo mi corazón y yo la curo porque quiero ver esa persona sana.

\subsubsection{Daños, malestares y padecimientos vinculados al trabajo en talleres textiles}

En el caso de las trabajadoras en talleres textiles de Buenos Aires, las distintas formas de violencia que experimentan en el marco de sus procesos migratorios van desde violencia estructural (Farmer, 2004); violencia física, sexual, psicológica, emocional y violencia simbólica, que dejan marcas en sus cuerpos y las exponen a una gravedad tal que pueden incluso llevarlas a la muerte. A su vez, en el espacio del taller-vivienda se ven expuestas a situaciones de riesgo que, por la interacción de un conjunto de factores, generan un proceso de desgaste de carácter permanente en su salud. Los ejemplos más explícitos y dramáticos de dichas situaciones de riesgo son los accidentes de trabajo, las enfermedades infecciosas como la TB; o los incendios de los recintos de trabajo/vivienda.

$\mathrm{Al}$ respecto, ha sido posible identificar una gran variedad de síntomas, signos, enfermedades, accidentes, malestares, aflicciones, padecimientos de distinto tipo y daños a la salud entre estas trabajadoras: daños a la vista, accidentes en dedos y manos, dolores continuos de espalda, hombros, nuca y cuello, neurosis, tensiones, nerviosismo, trastornos digestivos, problemas respiratorios (polvillo que despide la máquina de coser de manera constante, poca ventilación, hacinamiento), alergias, anemias, hipertensión arterial, etc. con una sobrecarga laboral intensa y por momentos ininterrumpi- 
da, todo lo cual impacta negativamente en su sistema psico-anímico, debilitando así su sistema inmunológico.

De acuerdo con las normas de higiene y seguridad laboral del Instituto Nacional de Tecnología Industrial, el corte de la tela con la máquina debería realizarse en un ámbito ventilado, utilizando guantes y barbijos (para prevenir la afectación de las vías respiratorias como consecuencia de la pelusa que se genera, y que se esparce por todo el ambiente), lo cual no sucede en la práctica. Respecto a la pérdida de la visión, a las condiciones ya mencionadas se le suma la poca iluminación que hay en el recinto del taller; un serio problema si se tiene en cuenta que el trabajo en la máquina requiere que el/ la trabajador/a fije permanentemente la vista en el corte. En el caso de las embarazadas, la intensidad del trabajo, sumada a la escasa y deficiente alimentación, ha producido riesgo fetal y para la vida de la mujer, cesáreas y abortos espontáneos, aparte de anemias crónicas. En consecuencia, enfermar y sufrir distintas dolencias y padecimientos en las condiciones de trabajo-vivienda descritas, resulta un hecho frecuente y recurrente para estas mujeres:

(...) al llegar al taller me dijeron que acá se trabajaba de 8 a 1 de la mañana (...) trabajé al mismo ritmo hasta el día que nació la criatura; me tuvieron que hacer cesáreas porque al estar sentada, el bebé no se había acomodado bien (...) Mis dos hijos nacieron alli (...) Luego me enfermé de anemia y tuve que dejar: en realidad, cuando el patrón se enteró que estaba enferma me empezó a "aborrecer". Quería que me fuera, pero no se animaba a echarme. Yo me fui, pero mi marido se quedó trabajando un tiempo más (Entrevista a $\mathrm{Bi}$, migrante boliviana, ex trabajadora en un taller textil clandestino de la CABA, integrante de la cooperativa textil "20 de diciembre-La Alameda").

Por su parte, el caso de Ni, entrevistada en sesiones múltiples en el pabellón Koch de internación para mujeres con TB del Hospital F. Muñiz de la CABA, grafica los riesgos de infección que supone el modo de vida-trabajo-vivienda en aquellos talleres, los cuales, para eludir los posibles controles e inspecciones, se localizan en villas o asentamientos de la ciudad de Buenos Aires, como la 1-11-14 del Bajo Flores. Por intermedio de una amiga que la contactó, esta mujer llegó al país "para trabajar en la costura" con su hermano, sin imaginar la situación en la que se encontraría una vez alcanzado el destino de su viaje y desconociendo absolutamente el contexto en el que estaba inserta: 
(...) Ahí [en el taller] había una persona que tenía tuberculosis.... Tosía mucho y de paso botaba mucha flema. Sí, y yo de seguro me contagié ahí porque había una pieza de cinco por cuatro, por ahí; ahi dormíamos cuatro personas... Pero no me di cuenta, no sabía de la enfermedad nada, no sabía bien el lugar, no sabía bien a dónde ir (...) Primero me dolía la espalda, luego me afectó el corazón, me dolía ahí, y no me di de cuenta que era tuberculosis, nada. Luego de un tiempo se pasó eso, no sentí nada. Luego tosí, tosí, tosí y boté flema con sangre. Ahí me trajo mi hermano aquí mismo -Hospital Muñiz-, y ahí me dijeron que tenía tuberculosis, que me tenía que internar (Entrevista a Ni, migrante boliviana, trabajadora en un taller textil clandestino de la CABA, internada con TB en el pabellón Koch de mujeres, Hospital Muñiz).

En la mayoría de los casos, a pesar de las dolencias, malestares y padecimientos sufridos por las trabajadoras, éstas continúan desarrollando sus tareas, tanto por miedo a las posibles represalias de los dueños del taller, como por la necesidad de producir para cobrar por prenda terminada. Del mismo modo, casos más extremos relevados, mostraron que los/las trabajadores/as tienen la prohibición de salir del recinto del taller debido a las condiciones, en muchos casos, análogas a la esclavitud, en las que se encuentran:

(...) Le decía: "me siento mal"; y ella [su tía, esposa del dueño del taller] me decía: "no sé qué tienes" y así... Y bueno, yo tenía miedo de decirle a ella, pero me dolía y le decía, y ella se ponía mal y me decía que, "bueno, hay que entregar —las prendas confeccionadas-. Yo seguía y seguía, en mi mente estaba "tengo que cumplir mi deuda, tengo que mandar [dinero a Bolivia], tengo que trabajar (Entrevista a Jr, migrante boliviana, ex trabajadora en un taller textil clandestino de la CABA).

Tenemos entonces que, a las situaciones de riesgo concretas en el trabajo y los ritmos productivos de éste, se le suman aquellas -muchas de las cuales son las mismas al encontrarse en el recinto del taller- ligadas a las condiciones de vivienda; y, adicionalmente, factores vinculados con las violencias de distinto tipo a las que son sometidas, la sobrecarga laboral, la deficiente alimentación y el estado emocional en el que se encuentran muchas de estas trabajadoras migrantes, presionadas para pagar deudas contraídas en origen. La interacción de estos factores de distinto tipo actúa como determinante en la baja general de su sistema inmunitario, lo cual las expone a la 
infección y/o el desarrollo de la TB, en un ambiente mal ventilado, donde las partículas portadoras de los bacilos de Koch pueden circular sin quedar expuestas a los efectos letales de la luz solar.

Finalmente, en el caso de las trabajadoras con sus hijos/as, deben adecuar sus tareas de cuidado con sus labores como costureras en el mismo recinto del taller-vivienda. Las/los niños/as pequeños/as, con frecuencia, no concurren al jardín de infantes o a la escuela. Son encerrados en algún rincón del taller, debiendo permanecer allí - por lo general, mirando la televisión-mientras la madre o el padre trabajan. En cuanto a los/as adolescentes, a partir de los 13 años son ubicados/as como aprendices del oficio de costura y paralelamente realizan otro tipo de actividades que obliga la dinámica productiva del taller (limpieza, doblado de telas y prendas, lavado, etc.). Al respecto, vale apuntar que los abusos y las agresiones contra las/los hijos por parte del tallerista, así como el sufrimiento de estas madres trabajadoras por no poder atender y cuidar de sus hijos/as como desean, constituye uno de los motivos más comunes para romper con lo que ellas denominan "el infierno de los talleres", y optar por insertarse en alguna de las cooperativas textiles existentes:

Cuando le tocaba al nene, al más chiquito, entrar al jardín no me dejaron llevarlo porque requería 15 minutos más de no producción y eso le afectaba y decía "no lo puedes meter, este año no, que vaya al otro año". Recién pudo ir al jardín cuando pude salir del taller (...) pero en lo que en mí fue un disparador, fue en una noche, una madrugada a las dos de la mañana, entre a mi cuarto y vi a mi hijo que estaba con un pan en la mano y un juguete en la otra, completamente frio, en la alfombra, que la habíamos entrado de la calle, y cuando yo lo toque pensé de que estaba muerto... eran las dos de la mañana (Entrevista a No, inmigrante boliviana, ex trabajadora en distintos talleres textiles clandestinos del AMBA).

\subsection{Discusión. Aproximación comparativa a los casos relatados}

Mediante las observaciones y entrevistas realizadas pudimos identificar, en el caso de las costureras bolivianas de Buenos Aires, sus experiencias subjetivas de haber sufrido violencias de distinto tipo o padecido TB; en cuanto a las trabajadoras agrícolas de Mendoza, identificamos la intensa carga de trabajo que suponen las condiciones de empleo en el agro y las dificultades en el acceso a la 
atención médica, cuya combinación produce diversos daños en su salud y padecimientos.

La inserción laboral de mujeres migrantes en fincas, fábricas y talleres se encuentra sujeta a distintas formas de precariedad. Un aspecto distintivo de las labores agrícolas es su inestabilidad, y la imprevisibilidad que esta genera en relación al sustento cotidiano. También la informalidad en el modo de contratación, que priva a las migrantes de la cobertura de seguridad social, resulta una problemática extendida en el sector. Sin embargo, estos aspectos se distancian de la situación de ilegalidad y trata laboral que organiza el trabajo en talleres textiles, que empujan a las mujeres migrantes a situaciones próximas a la esclavitud.

En contraste con estas diferencias, encontramos coincidencias en los espacios de empleo mencionados: en fincas y talleres se repite el pago a destajo, la superposición entre lugar de trabajo y lugar de vivienda, y la doble jornada. El primero redunda en jornadas laborales extensas, que aumentan el desgaste laboral y limitan las posibilidades de recuperarse. La intensificación del esfuerzo y el empleo de la fuerza de trabajo familiar (incluidos/as sus hijos/ as) también resulta una consecuencia directa de esta forma de remuneración. El segundo, referido a la convergencia entre lugar de vivienda y trabajo, constituye uno de los rasgos que expone a las trabajadoras a las condiciones de reproducción más críticas: se alimentan, duermen y cuidan de sus hijos/as en espacios reducidos, mal ventilados o escasamente protegidos de inclemencias climáticas; en ocasiones carecen de camas y baños; y sufren restricciones para circular libremente, sea por la ubicación de las fincas situadas en extensas superficies que sólo es posible recorrer en vehículos, o por la explícita prohibición de salir de los talleres. El tercer aspecto, la sumatoria del trabajo que realizan en talleres textiles, fábricas y fincas con sus labores domésticas/reproductivas, crea una "doble carga" que supone diversos malestares y padecimientos, los cuales suelen ser invisibles para marcos interpretativos androcéntricos, que no consideran trabajo a las actividades reproductivas.

El control del proceso de trabajo también se presenta como un rasgo próximo entre talleres textiles y fábricas de empaque de frutas, por cuanto las actividades laborales de las mujeres se ven estrictamente vigiladas. Sin embargo, también en este punto se aprecian diferencias, dado que la situación de las trabajadoras en los talleres se ve agravada por el encierro y la prohibición de salir. 
Las experiencias en torno a la salud-enfermedad-cuidados que recuperamos denotan no sólo los daños, enfermedades y accidentes vinculados al trabajo en talleres, fincas, y fábricas; sino que también evidencian el agotamiento y malestar subjetivo que sobreviene cuando el empleo se tensiona con el descanso, el cuidado de la propia salud y la de sus grupos o la libertad de movimiento. Estos espacios laborales constituyen lugares que marcan el cuerpo (Csordas, 1994) de sus trabajadoras mediante intoxicaciones, quemaduras dérmicas y problemas respiratorios asociados a las condiciones de labor. Pero además suscitan padecimientos por la estricta vigilancia e, incluso, la violencia que posibilita la explotación de las trabajadoras migrantes.

Con diferencias específicas en cada caso, visualizamos que las condiciones de trabajo/vivienda constituyen un obstáculo para que las trabajadoras accedan a la atención médica cuando lo requieren. Esto torna relevantes a las estrategias cotidianas de cuidado de la salud: desde prácticas alimentarias vinculadas a sus contextos de origen, hasta el ejercicio de medicina campesina, las migrantes ponen en práctica diversas formas de cuidado alternativas a la biomedicina. De este modo, no sólo sortean el limitado acceso a los servicios biomédicos, sino que también posibilitan la persistencia de concepciones sobre el cuerpo y la salud vinculadas con sus trayectorias vitales. Entre las estrategias de cuidado mencionadas no podemos soslayar aquellas que, sin constituir una práctica de atención de los padecimientos en sentido estricto, implican la posibilidad de preservar la vida y la integridad física de las migrantes y sus familias: salir del infierno de los talleres. Ello nos indica que, en condiciones extremas como las de los talleres textiles, preservar la salud sólo es posible si se abandona el trabajo y, como lo demuestra este estudio, también la vivienda.

\section{CONCLUSIONES}

Este artículo buscó realizar un aporte, en clave comparativa, a la relativa escasez de producciones existentes desde las Ciencias Sociales en nuestro país sobre la salud y los procesos migratorios internacionales contemporáneos. A través del análisis comparativo de dos casos de estudio etnográficos ubicados en localidades argentinas diferentes, nos propusimos explicar la manera en que 
opera la dialéctica entre desigualdades sexo-genéricas, de clase, vinculadas al estatus migratorio, y las desigualdades en salud; esto es, la forma en que determinados contextos de vulnerabilidad social se expresan en indicadores diferenciales en cuanto a las condiciones de acceso a los servicios públicos de salud y a las enfermedades y padecimientos experimentados, su diagnóstico y tratamiento (Goldberg, 2014b).

La contribución específica del abordaje propuesto consistió en evidenciar, a través de herramientas conceptuales feministas y de la antropología de la salud, pero fundamentalmente a partir de las narrativas de las trabajadoras, la complejidad de las experiencias de salud/enfermedad/cuidado de un grupo de mujeres migrantes en Argentina. Los relatos visibilizan problemáticas diversas: enfermedades laborales producidas por las condiciones de empleo, inaccesibilidad a servicios de atención médica, múltiples padecimientos vinculados con modos de vida-vivienda-trabajo precarizados. En suma, las experiencias aportan a comprender el modo en que la reproducción cotidiana en contextos de vulnerabilidad social se imprime en el cuerpo, la subjetividad y la salud de las migrantes.

Cabe señalar algunas limitaciones del enfoque propuesto: en el plano metodológico, es posible mencionar las dificultades y obstáculos que supone desarrollar trabajo de campo en espacios clandestinos y/o inaccesibles, que restringen la recopilación de información. En el plano analítico, por lo inicial del planteo realizado, probablemente se requiera avanzar en la identificación de la variabilidad, diversidad y heterogeneidad de los distintos grupos, a fin de evitar esquemas de análisis comparativos generalizadores forzados.

Para finalizar, consideramos que algunas de las temáticas aquí planteadas de forma incipiente, invitan a profundizar indagaciones comparativas alrededor del fenómeno contemporáneo de la explotación laboral de mujeres migrantes en los distintos mercados precarios generados por el capitalismo global, y el impacto que dichas condiciones tienen en su salud. Al mismo tiempo, resulta necesario ahondar en el estudio de los saberes y prácticas que desarrollan para hacer frente a esos problemas, como forma de evitar miradas victimizantes sobre las mujeres y para destacar sus posibilidades de resistir, afrontar y modificar aquellas situaciones que las aquejan. 


\section{BIBLIOGRAFÍA}

Álvarez, C. (2011). El interés de la etnografía escolar en la investigación educativa. Estudios pedagógicos (Valdivia), 37(2), 267-279.

Aparicio, S. (2012). Caso Argentina. En F. Soto Baquero, \& E. Klein, Empleo y condiciones de trabajo de mujeres temporeras agrícolas (págs. 11-141). CEPAL, OIT, FAO.

Arizpe, L., \& Aranda, J. (1981). El empleo agroindustrial y la participación de la mujer en el desarrollo rural: un estudio de las obreras del cultivo de exportación de la fresa en Zamora, México. Michoacán: Organización Internacional del Trabajo.

Benencia, R. (2009). Inserción de bolivianos en el mercado de trabajo de la Argentina. Congreso 2009 de la Asociación de Estudios Latinoamericanos. Río de Janeiro.

Benencia, R. (2009a). El infierno del trabajo esclavo: La contracara de las' exitosas' economías étnicas. Avá, (15), 00-00.

Benería, L. (1981). Reproducción, producción y división sexual del trabajo. Mientras Tanto (6), 47-84.

Brito, J. C., Neves, M. Y., Oliveira, S. S., \& Rotenberg, L. (2012). Saúde, subjetividade e trabalho: o enfoque clínico e de gênero. Revista Brasileira de Saúde Ocupacional, 37(126), 316-329.

Burín, M. (1990). El malestar de las mujeres. La tranquilidad recetada. Buenos Aires: Paidós.

Camarena Ojinaga, L., von Glascoe, C., Martínez Valdés, C., \& Arellano García, E. (2013). Occupational risks and health: perceptions of indigenous female agricultural workers in Northwestern Mexico. Salud Colectiva, 9 (2), 247-256.

Carrasco, C. (2011). La economía del cuidado: Planteamiento actual y Desafíos pendientes. Revista de Economía Crítica (11), 205-225.

Crenshaw, K. W. (2012). Cartografiando los márgenes: Interseccionalidad, políticas identitarias y violencia contra las mujeres de color. En Intersecciones: cuerpos y sexualidades en la encrucijada (págs. 87-122). Madrid: Bellaterra.

Csordas, T. (1994). Embodiment and Experience. Cambridge: Cambridge University Press.

Davis, A. (2005 [1981]). Mujeres, raza y clase. Madrid: Akal.

Esteban, M. L. (2010). Diagnósticos en salud y género: aportaciones antropológicas para una perspectiva integral de análisis. En Esteban, M. L., Comelles, J. M., Díez Mintegui, C. Antropología, género, salud y atención (p. 49-68). Barcelona: Bellaterra.

Farmer, P. (2004). An Anthropology of Structural Violence. Current Anthropology, (45), 305-325.

Federici, S. (2013). Revolución en punto cero. Trabajo doméstico, reproducción y luchas feministas. Madrid: Traficantes de Sueños. 
Goldberg, A. (2010). Análisis de la relevancia de los factores socioculturales en el proceso asistencial de pacientes con tuberculosis, usuarios del Instituto Vaccarezza - Hospital Muñiz. Un abordaje etnográfico desde la Antropología Médica. Revista Argentina de Salud Pública, 1 (5), 13-21.

Goldberg, A. (2014a). Trayectorias migratorias, itinerarios de salud y experiencias de participación política de mujeres migrantes bolivianas que trabajaron y vivieron en talleres textiles clandestinos del Área Metropolitana de Buenos Aires, Argentina. Anuario Americanista Europeo, (11), 199-216.

Goldberg, A. (2014b). Contextos de vulnerabilidad social y situaciones de riesgo para la salud: tuberculosis en inmigrantes bolivianos que trabajan y viven en talleres textiles clandestinos de Buenos Aires. Cuadernos de Antropología Social (39), 91-114.

Goldberg, A. (2016). La parte invisibilizada de la migración transnacional boliviana hacia Argentina. Andamios, 32 (13), 357-378.

Grimberg, M. (2009). Experiencias y narrativas de padecimientos cotidianos. Miradas antropológicas sobre la salud, la enfermedad y el dolor crónico. Buenos Aires: Antropofagia.

Klein, N. (2001). No Logo, El poder de las marcas. Buenos Aires: Paidós.

Krieger, N. (2003). Genders, sexes, and health: what are the connections and why does it matter? International Journal of Epidemiology, (32), 652-657.

Lara Flores, S. M. (1995). La feminización del trabajo asalariado en los cultivos de exportación no tradicionales en América Latina: efectos de una flexibilidad "salvaje". En S. M. Lara Flores, Jornaleras, temporeras y bóias frías. El rostro femenino del mercado de trabajo rural en América Latina (págs. 13-34). Caracas: Nueva Sociedad.

Lara Flores, S. M. (2003). Violencia y contrapoder: una ventana al mundo de las mujeres indígenas migrantes, en México. Estudios Feministas, 11 (2), 381-397.

Lieutier, A. (2010). Esclavos. Los trabajadores costureros de la ciudad de Buenos Aires. Buenos Aires: Retórica Ediciones.

Magliano, M. J. (2009). Migración, género y desigualdad social. La migración de mujeres bolivianas hacia Argentina. Estudios Feministas, 17 (2), 349-367.

Magliano, M. J., \& Domenech, E. E. (2009). Género, política y migración en la agenda global: Transformaciones recientes en la región Sudamericana. Migración y desarrollo, (12), 53-68.

Mallimaci, F., \& Giménez Béliveau, V. (2006). Historia de vida y métodos biográficos. En I. Vasilachis de Gialdino, Estrategias de investigación cualitativa (págs. 175-212). Barcelona: Gedisa.

Maure, G., Linardelli, M. F., \& Anzorena, C. (2016). ¿Autoatención o trabajo de cuidados? El rol de mujeres migrantes bolivianas en el cuidado de familiares enfermos/as. Jornadas de Antropología Social Santiago Wallace. Buenos Aires: Universidad de Buenos Aires - Instituto de Ciencias Antropológicas. 
Maxwell, J. A., \& Miller, B. A. (2008). Categorizing and connecting strategies in qualitative data analysis. En P. Leavy, \& S. Hesse-Biber, Handbook of emergent methods (págs. 461-477). New York: Guilford Press.

Menéndez, E. (2005). Intencionalidad, experiencia y función: la articulación de los saberes médicos. Revista de Antropología Social, 33-69.

Mingo, E. (2015). Resistentes, comprometidas y conflictivas: obreras de la agroindustria frutícola en Argentina. Una mirada desde la demanda de mano de obra. En A. Riella, \& P. Mascheroni, Asalariados rurales en América Latina (págs. 95-110). Montevideo: CLACSO.

Miranda, B. (2017). "Uno ya sabe a lo que viene": la movilidad laboral de migrantes andino-bolivianos entre talleres de costura de São Paulo explicada a la luz de la producción del consentimiento. Remhu-Revista Interdisciplinar da Mobilidade Humana, 25(49).

Moreno, M. S. (2017). De pasaditas no más voy. La participación de los migrantes bolivianos en las cosechas agrícolas de Mendoza. Estudio de caso a partir de una etnografía multilocal. Tesis doctoral en ciencias sociales. Mendoza: Universidad Nacional de Cuyo.

Moreno, M., \& Torres, L. (2013). Movimientos territoriales y dinámicas laborales: los migrantes bolivianos en la agricultura de Mendoza (Argentina). CRITERIOS - Cuadernos de Ciencias Jurídicas y Política Internacional, 6(1), 19-58.

Moreno, M. S., \& Martínez Espínola, M. V. (2017). Trayectorias de mujeres bolivianas en áreas rurales y urbanas de Mendoza (Argentina). Si Somos americanos. Revista de Estudios Transfronterizos, 73-99.

Pérez Orozco, A. (2014). Del trabajo doméstico al trabajo de cuidados. En Cristina Carrasco(ed), Con voz propia. La economía feminista como apuesta teórica y política (págs. 49-74). Madrid: La Oveja Roja.

Pessolano, D. (2016). Economía de la vida. Aportes de estudios feministas y de género. Polis, Revista Latinoamericana, 15(45), 191-209.

Rodríguez Enríquez, C. (2012). La cuestión del cuidado: ¿El eslabón perdido del análisis económico? Revista CEPAL (106), 23-36.

Salgado, P. D. (2015). Deslocalización de la producción y la fuerza de trabajo: Bolivia-Argentina y las tendencias mundiales en la confección de indumentaria. Si Somos americanos, 15(1), 169-198.

Scheper-Hughes, N., \& Lock, M. M. (1987). The mindful body: A prolegomenon to future work in medical anthropology. Medical anthropology quarterly, 1(1), 6-41.

Simbiosis Cultural \& Colectivo Situaciones. (2011). De chuequistas y overlockas. Una discusión en torno a los talleres textiles. Buenos Aires: Tinta Limón.

Tajer, D., Reid, G., Gaba, M., Lo Russo, A., y Barrera, M. I. (2013). Investigaciones sobre género y determinación psicosocial de la vulnerabilidad coronaria en varones y mujeres. Revista Argentina de Cardiología, (81), 344-352. 
Teubal, M. (2001). Globalización y nueva ruralidad en América Latina. En N. Giarraca, ¿Una nueva ruralidad en América Latina? Buenos Aires: CLACSO.

Valdés Subercaseaux, X. (2015). Feminización del empleo y trabajo precario en las agriculturas latinoamericanas globalizadas. Cuadernos de Antropología Social (41), 39-54.

Valls-Llobet, C. (2009). Mujeres, salud y poder. Madrid: Ediciones Cátedra.

Velasco, S., Ruiz, M ${ }^{\mathrm{a}}$ T., \& Álvarez-Dardet, C. (2006). Attention Models to Somatic Symptoms without Organic Cause: From Phisio-pathologic Disorders to Malaise of Women. Revista Española de Salud Pública, 80(4), 317-333. 